\title{
An extraordinary origami curve
}

\author{
Frank Herrlich ${ }^{*}$ Gabriela Schmithüsen ${ }^{\dagger}$ \\ Mathematisches Institut II, Universität Karlsruhe, 76128 Karlsruhe, Germany
}

\begin{abstract}
We study the origami $W$ defined by the quaternion group of order 8 and its Teichmüller curve $C(W)$ in the moduli space $M_{3}$. We prove that $W$ has Veech group $\mathrm{SL}_{2}(\mathbb{Z})$, determine the equation of the family over $C(W)$ and find several further properties. As main result we obtain infinitely many origami curves in $M_{3}$ that intersect $C(W)$. We present a combinatorial description of these origamis.
\end{abstract}

Origami curves are certain special Teichmüller curves in some moduli space of curves. They are obtained from an unramified covering of a once punctured torus, see Section 1.1 for a precise definition.

First examples of Teichmüller curves were already given by Veech in V]. In recent years they have attracted a lot of attention, partly because of their relation to rational billiards, see e.g. $[\mathrm{McM}]$ and references therein; a survey of examples defining primitive Teichmüller curves can be found in $\mathrm{HuSc}$. Another topic of interest is the action of the absolute Galois group of the rationals on them as discussed in [LO and [M1].

In some respects Teichmüller curves arising via origamis are more accessible than general ones. Precisely for them, the Veech group (as defined in Section 1.1) is a subgroup of $\mathrm{SL}_{2}(\mathbb{Z})$, cf. GJ]. It can be determined for each origami explicitly, cf. [S]. Nevertheless, it is still difficult to approach the question how their Teichmüller curves are located in the moduli space. There are only a few origami curves for which explicit equations have been found so far, cf. [H] and [M1].

In this note we present an extraordinary origami curve in genus 3 . It is the smallest nontrivial example of a normal origami having as Veech group the full group $\mathrm{SL}_{2}(\mathbb{Z})$, see Prop. 2 The Jacobian of the associated family of curves has a two-dimensional fixed part, see Proposition 7 M. Möller has observed that this implies that our origami curve is also a Shimura curve; recently he proved that it is the only algebraic curve in a moduli space of curves of genus at least 2 which is at the same time a Teichmüller curve and a Shimura curve, cf. [M2]. An explicit equation for the associated family of curves is given in Proposition 5 This family of curves has been studied by several authors, and some of the results of Section 1 have been known previously, cf. Gi], Gu2], [KK]; nevertheless the use of the "origami" structure allows for new proofs and makes the exposition considerably more elementary.

A very remarkable property of this origami curve, and the main new result of this paper, is the fact that it intersects infinitely many other origami curves, see Theorem 1 in Section 3 To our knowledge this is the first example of origami curves that intersect in moduli space.

The decomposition of the Jacobian gives a second map onto an elliptic curve; if this map is ramified only over torsion points it can be made into an origami. In Section 2 we develop explicit formulas for these maps to determine when this particular type of ramification occurs.

We give a combinatorial description of the infinitely many origamis that intersect our origami

*e-mail: herrlich@math.uni-karlsruhe.de,

$\dagger$ e-mail: schmithuesen@math.uni-karlsruhe.de 
curve, see Proposition 16 in Section 3.2 They can be obtained by suitably glueing two copies of an $n \times n$-square (for different $n$ ).

Acknowledgement: The results in the first section were obtained together with Martin Möller. We would like to thank him for allowing us to include this part. When he visited Karlsruhe and we discovered one remarkable property of this origami after the other, we called it "eierlegende Wollmilchsau". We still call it $W$ in this paper.

\section{The origami $W$}

We start in 1.1 with a brief introduction to origamis. In 1.2 we introduce the origami $W$, to which the title of this paper refers, and prove that it has Veech group $\mathrm{SL}_{2}(\mathbb{Z})$. In 1.3 1.6 we examine properties of $W$ : We describe its automorphism group (1.3), give algebraic equations for the corresponding family of curves (1.4), find the Jacobian (1.5) and determine the only cusp of its origami curve (1.6).

\subsection{Origamis and their Veech groups}

Here we state the basic definitions and facts that we will use in this article. We follow mostly the notations for origamis used in $\underline{\underline{S}}$. A more detailed introduction to translation surfaces and Veech groups can be found e.g. in GJ and [HuSc. Some interesting facts about origamis are contained e.g. in [HL and [M1. For an introduction to Teichmüller geodesics and Teichmüller curves we refer to $[\mathrm{EG}]$ and $[\mathrm{McM}]$.

An origami is a finite covering $p: X \rightarrow E$ from a closed surface $X$ to a torus $E$ which is unramified over $E^{\star}:=E-\{0\}$, where $0 \in E$ is a point. Any complex uniformization $E_{\tau}=$ $\mathbb{C} /(\mathbb{Z}+\tau \mathbb{Z})$ of $E$ defines a translation structure on $E$ which lifts to a translation structure on $X^{\star}:=p^{-1}\left(E^{\star}\right)=X-p^{-1}(0)$ (therefore an origami is also called a square tiled surface). The translation structure is also obtained from the holomorphic 1-form $\omega_{\tau}:=p^{*} \omega_{E_{\tau}}$, where $\omega_{E_{\tau}}$ is the invariant differential on the elliptic curve $E_{\tau}$. If $X_{\tau}$ denotes the complex structure on $X$ determined by this translation structure, the map $\tau \mapsto X_{\tau}$ is an isometric embedding of the upper half plane into the Teichmüller space $T_{g, n}$ (where $g$ is the genus of $X$ and $\left.n=\left|p^{-1}(0)\right|\right)$; such embeddings are called Teichmüller geodesics or Teichmüller disks.

Conversely, any finite collection of euclidean squares together with a pairing that glues every left edge to a right one and every top edge to a bottom one, uniquely determines an origami. It is this combinatorial construction that inspired P. Lochak in [Lo to use the name "origami". In the sequel we shall describe origamis usually in this way.

For an origami, and more generally for a translation surface $X^{\star}$ of finite volume, the Veech group $\Gamma\left(X^{\star}\right) \subset \mathrm{SL}_{2}(\mathbb{R})$ is defined as follows: Let $\mathrm{Aff}^{+}\left(X^{\star}\right)$ be the group of orientation preserving diffeomorphisms of $X^{\star}$ which are affine with respect to the given translation structure. The linear part of $\varphi \in \operatorname{Aff}^{+}\left(X^{\star}\right)$ is the same on every chart and gives rise to a group homomorphism der: $\mathrm{Aff}^{+}\left(X^{\star}\right) \rightarrow \mathrm{SL}_{2}(\mathbb{R})$; then $\Gamma\left(X^{\star}\right)$ is defined as its image. It is a discrete subgroup of $\mathrm{SL}_{2}(\mathbb{R})$, see $[\mathrm{V}]$.

The translation surfaces corresponding to the points on a Teichmüller disk are related by affine diffeomorphisms. Their Veech groups are conjugated in $\mathrm{SL}_{2}(\mathbb{R})$ and it is therefore sufficient to consider the Veech group of a base point. In the special case of an origami $O$ we choose $\tau=i$ and denote $\Gamma(O):=\Gamma\left(O_{i}\right)$. It is known that this is a subgroup of $\mathrm{SL}_{2}(\mathbb{Z})$ of finite index (see $[\underline{S}$ for a proof and also for more details about origamis and Veech groups). Therefore $\Gamma(O)$ acts on the upper half plane $\mathbb{H}$ as a lattice, and the orbit space $\mathbb{H} / \Gamma(O)$ is a nonsingular affine curve. It is the normalization of the image $C(O)$ of the Teichmüller geodesic defined by $O$ in the moduli space $M_{g, n}$. The algebraic curve $C(O)$ is called the origami curve associated with $O$. This construction is a special case of the more general concept of a Teichmüller curve. 


\subsection{The quaternion origami}

The origami $W$, which is the central object of this paper, can be described as follows: Let $Q:=\{ \pm 1, \pm i, \pm j, \pm k\}$ be the quaternion group. Recall that the (defining) relations are $i^{2}=j^{2}=k^{2}=-1, i j=-j i=k,(-1)^{2}=1$. Thus $i k=-k i=-j$ and $j k=-k j=i$. Take eight squares labeled with the elements of $Q$ and glue them in horizontal (resp. vertical) direction such that the right neighbour of the square labeled $g$ has label $g \cdot i$ and its top neighbour has label $g \cdot j$. The resulting origami $W$ can be represented as
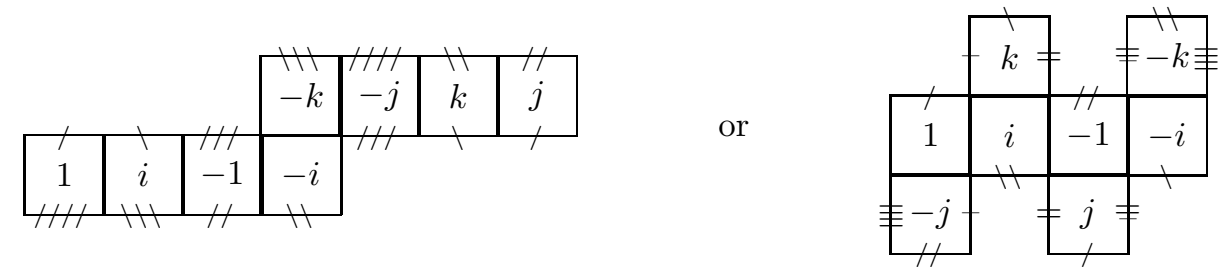

Figure 1

Edges are glued if they have the same label or are opposite and unlabeled.

The commutator of the generators is $i j(-i)(-j)=-1$, thus of order 2. This implies that every vertex belongs to 8 squares. Therefore the number of vertices on $W$ is $(8 \cdot 4): 8=4$. The Euler formula gives $2-2 g=8-16+4=-4$, so the genus of $W$ is 3 .

Remark 1. The origami map $p: W \rightarrow E$, which maps each of the eight squares to the standard torus $E$, is a normal covering with Galois group $Q$. The elements of $Q$ act as translation automorphisms on $W$ by left(!) multiplication on the labels of the squares.

This follows from the fact that Figure 1 can also be seen as the Cayley graph of $Q$ with respect to the generators $i$ and $j$ (taking the squares as vertices and the glueings as edges).

Let $p: W^{\star} \rightarrow E^{\star}$ be the unramified covering induced by $W$ (i. e. $W^{\star}=W-p^{-1}(0)$ ). By the theorem of the universal covering it defines an embedding of $H:=\pi_{1}\left(W^{\star}\right)$ into $\pi_{1}\left(E^{\star}\right) \cong F_{2}$, the free group on two generators. We choose as generators the horizontal and vertical simple closed curves $x$ and $y$ on $E^{\star}$. If $\alpha: F_{2} \rightarrow Q$ denotes the homomorphism that maps $x$ to $i$ and $y$ to $j$, then $H$ is the kernel of $\alpha$.

Proposition 2. (i) $H$ is a characteristic subgroup of $F_{2}$.

(ii) The Veech group $\Gamma(W)$ of $W$ is $\mathrm{SL}_{2}(\mathbb{Z})$.

Proof. The second statement follows from the first by [S, Prop. 1].

To show that $H=\operatorname{ker}(\alpha)$ is preserved by all automorphisms of $F_{2}$ we shall show that every surjective homomorphism $\beta: F_{2} \rightarrow Q$ has kernel equal to $H$. For this it suffices to observe that $\beta$ is obtained from $\alpha$ by composition with an automorphism of $Q$ : Let $a:=\beta(x)$ and $b:=\beta(y)$; since $\beta$ is surjective, $a$ and $b$ are elements of order 4 satisfying $a \neq b \neq-a$. Then the map $i \mapsto a, j \mapsto b$ respects all the defining relations of $Q$, hence is an automorphism $\sigma$ of $Q$; by construction, $\beta=\sigma \circ \alpha$.

For a more systematic approach to finding such characteristic origamis $\mathrm{cf}$. $\mathrm{H}$.

Proposition 3. $W /\{ \pm 1\}$ is isomorphic to $E$ and the origami map $p: W \rightarrow E$ is the composition of the quotient map and multiplication by 2.

Proof. Note that $-1=i^{2}=j^{2}$ acts on the squares of the origami by translation by 2 in both horizontal and vertical direction. In particular, -1 fixes the four punctures of $W$, as can be read off from the picture (note that the four vertices of each square all belong to different punctures on $W$ ). By the Riemann-Hurwitz formula this implies that $W /\{ \pm 1\}$ is an elliptic curve.

More precisely, $W /\{ \pm 1\}$ is the origami 


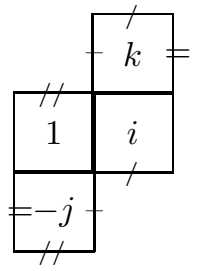

which is equivalent to

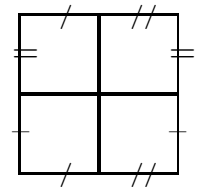

Figure 2

This shows that the origami map $p: W \rightarrow E$ is the composition of the quotient map $W \rightarrow W /\{ \pm 1\}$ and multiplication by 2 .

The second step also follows from the fact that $Q /\{ \pm 1\}$ is isomorphic to the Klein four group $V_{4}$.

\subsection{The automorphism group of $W$}

We shall examine the group $G:=\operatorname{Aut}(W)$ of affine holomorphic automorphisms living on all translation structures defined by $W$. Any such automorphism descends to an affine map on the elliptic curve with derivative $I$ or $-I$, where $I$ is the identity matrix (see e.g. $[\underline{S}$ ). Obviously, $Q \subseteq G$ contains the automorphisms with derivative $I$ (compare 1.2). In this section we give the group structure of the whole group $\operatorname{Aut}(W)$.

$W$ admits a further automorphism $\sigma$ that is not in $Q$ : the canonical involution (i.e. multiplication by -1) on $E$ can be lifted to $W$. This follows from the fact that $-I$ is in the Veech group of $W$. Explicitly, we describe $\sigma$ by rotating each square around its center, and then glueing the same edges as before:

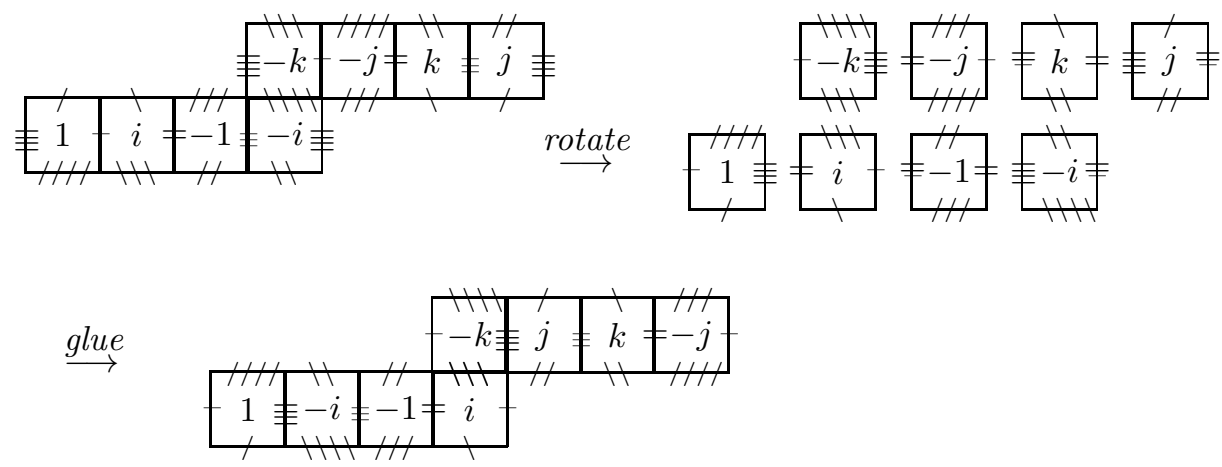

Figure 3

The fixed points of $\sigma$ are the centers of the squares with labels $1,-1, k$, and $-k$. So $\sigma$ can also be described as rotation of $W$ around the center of (one of) these squares.

Again by Riemann-Hurwitz we find that $W /\langle\sigma\rangle$ has genus 1. We shall determine this elliptic curve below in Section 1.5 .

From the picture of the origami we see that $\tau:=i \sigma$ has order 2 , and that the same holds for $\rho:=j \sigma$. This implies $i \sigma(-i)=-\sigma$ and $j \sigma(-j)=-\sigma$. As a consequence, $\sigma$ commutes with $k$. The element $c:=k \sigma$ therefore satisfies

$$
c^{2}=(k \sigma)^{2}=k \sigma \sigma k=k^{2}=-1 .
$$

In particular, $c$ is of order 4; it commutes with $k$ and $\sigma$, and also with $i$ and $j$, thus it is in the center of $G$. With this information at hand we have determined the group structure of $G$ :

Proposition 4. a) The group $G=\operatorname{Aut}(W)$ is generated by $Q$ and $\sigma$ and has order 16 . It consists of eight elements of order 4 , namely $\pm i, \pm j, \pm k$ and $\pm c$, and seven elements of order 2, namely -1 and $\pm \sigma, \pm \tau$ and $\pm \rho$. In the list of Hall and Senior [HaSe] it belongs to the family $\Gamma_{2}$ and is number 8 among the groups of order 16 . 
b) The automorphisms in $Q$ descend via $p$ to the identity on $E$, whereas those in $G \backslash Q$ descend to involutions. On the degree 2 quotient $W /\{ \pm 1\} \cong E$ (see Proposition (3) the elements of $Q$ induce translations and those in $G \backslash Q$ induce involutions.

c) The elements of $G$ have the following fixed points in $W$ :

$\pm i, \pm j$ and $\pm k$ have no fixed points.

$\sigma$ fixes the centers of the squares $1,-1, k,-k$.

$-\sigma$ fixes the centers of the squares $i,-i, j,-j$.

$\tau$ fixes the centers of the vertical edges between 1 and $i,-1$ and $-i,-j$ and $k, j$ and $-k$.

$-\tau$ fixes the centers of the remaining vertical edges.

$\rho$ fixes the centers of the horizontal edges between 1 and $j, i$ and $k,-1$ and $-j,-i$ and $-k$.

$-\rho$ fixes the centers of the remaining horizontal edges.

$c$ (and hence also -1) fixes the vertices of the squares.

\subsection{The origami curve $C(W)$}

By Proposition 4 $C(W)$ is a 1-parameter family of curves of genus 3 with automorphism group $G$. This uniquely determines this family, see $[\mathrm{KK}$. Using the origami structure we can give an independent direct proof:

Proposition 5. a) The origami curve $C(W)$ is the image in $M_{3}$ of the 1-parameter family of smooth plane curves $W_{\lambda}$ with affine equation

$$
y^{4}=x(x-1)(x-\lambda) \quad \text { with } \lambda \in \dddot{\mathbb{P}}:=\mathbb{P}^{1}(\mathbb{C})-\{0,1, \infty\}
$$

The quotient map $W_{\lambda} \rightarrow W_{\lambda} /\{ \pm 1\}=E_{\lambda}: y^{2}=x(x-1)(x-\lambda)$ is given by

$$
(x, y) \mapsto\left(x, y^{2}\right) .
$$

b) $C(W)$ is nonsingular and isomorphic to $\mathbb{H} / \Gamma(W)=\mathbb{A}^{1}$.

Proof. a) From the Riemann-Hurwitz formula and the fact that $c$ has four fixed points on $W$ we conclude $4=4 \cdot(2 g(W /\langle c\rangle)-2)+4 \cdot 3$ which implies that $W /\langle c\rangle$ is a rational curve. Thus $W$ is a cyclic covering of order 4 of the projective line, totally ramified over four distinct points. Normalizing three of them to 0,1 and $\infty$ we see that $W$ has the equation

$$
y^{4}=x^{\varepsilon_{0}}(x-1)^{\varepsilon_{1}}(x-\lambda)^{\varepsilon_{\lambda}},
$$

where the parameter $\lambda$ is different from 0,1 and $\infty$ and depends on (or rather determines) the complex structure of $W$, and the exponents $\varepsilon_{i}$ can be 1 or 3 (2 is excluded because the covering is totally ramified over all four points). Replacing $y$ by $y^{-1} x(x-1)(x-\lambda)$ if $\varepsilon_{0}=3$ we may assume $\varepsilon_{0}=1$. The values of $\varepsilon_{1}$ and $\varepsilon_{\lambda}$ are determined by the monodromy action of $c$ on the loops around 0,1 and $\lambda$ : from the origami we read off that $c$ acts by counterclockwise rotation at all four fixed points. This implies that the exponent is the same at all ramification points and thus proves the first statement.

The map given in the claim is compatible with the automorphism -1 of $W_{\lambda}$, which is $-1:(x, y) \mapsto(x,-y)$. Therefore it is the quotient map.

b) From Proposition 2 we know that the Veech group $\Gamma(W)$ is $\mathrm{SL}_{2}(\mathbb{Z})$, thus $\mathbb{H} / \Gamma(W)$ is isomorphic to the affine line. On the other hand, $C(W)$ is the image of the map $\dddot{\mathbb{P}} \rightarrow M_{3}$ that sends $\lambda$ to the isomorphism class of the plane quartic $W_{\lambda}$. Since an embedding of a genus three curve into the projective plane is necessarily the canonical embedding, two such curves are isomorphic as abstract curves if and only if they are isomorphic as embedded curves. This means that for $\lambda, \lambda^{\prime} \in \dddot{\mathbb{P}}, W_{\lambda}$ and $W_{\lambda^{\prime}}$ define the same point in $M_{3}$ if and only if there is a projective change of coordinates that maps $W_{\lambda}$ onto $W_{\lambda^{\prime}}$. As in the case of the Legendre family $y^{2}=x(x-1)(x-\lambda)$ of elliptic curves such a projectivity exists if and only if $\lambda^{\prime}$ is obtained from $\lambda$ by a Möbius transformation that permutes the three points $0,1, \infty$. This shows that $C(W)$ is isomorphic to $\dddot{\mathbb{P}} / S_{3} \cong \mathbb{A}^{1}$. 


\subsection{The Jacobian of $W$}

We first determine the quotient of $W$ by $\sigma$ : Since $\sigma$ commutes with $c$ but is not in the center, the normalizer of $\sigma$ in $G$ is a group of order 8 , isomorphic to $\mathbb{Z} / 2 \mathbb{Z} \times \mathbb{Z} / 4 \mathbb{Z}$. This shows that $c$ descends to an automorphism $\bar{c}$ of order 4 on the elliptic curve $E_{\sigma}:=W /\langle\sigma\rangle$. Since $\bar{c}$ has a fixed point (see Proposition 4), it is not a translation. The only elliptic curve with such an automorphism is $E_{-1}$ which has equation $y^{2}=x^{3}-x$ and $j$-invariant 1728 . By symmetry the same reasoning also holds for the other involutions:

Proposition 6. For any $\lambda \in \dddot{\mathbb{P}}$, the quotient of $W_{\lambda}$ by each of the involutions $\pm \sigma, \pm \tau$ and $\pm \rho$ is the elliptic curve $E_{-1}$.

The following result is contained in Gu1, Thm. 7.3], where also the degree of the isogeny is determined. It is also a special case of a much more general result in $\mathrm{W}$. We include an elementary proof which makes essential use of the origami structure.

Proposition 7. For every $\lambda \in \dddot{\mathbb{P}}$, the Jacobian of $W_{\lambda}$ is isogenous to $E_{\lambda} \times E_{-1} \times E_{-1}$.

Proof. Fix a quotient morphism $\varphi: W_{\lambda} \rightarrow W_{\lambda} /<\sigma>\cong E_{-1}=: E$. Since $-\sigma=i \sigma(-i)$ we see that $\psi:=\varphi \circ i: W_{\lambda} \rightarrow E$ is a quotient map for the action of $-\sigma$.

Let $\beta$ : Jac $W_{\lambda} \rightarrow E \times E$ be the homomorphism (of abelian varieties) induced by $(\varphi, \psi)$ : $W_{\lambda} \rightarrow E \times E$. We claim that $\beta$ is surjective. Together with the quotient map $W_{\lambda} \rightarrow$ $W_{\lambda} /\{ \pm 1\}=E_{\lambda}$ from Proposition 3 we then obtain the desired isogeny.

To prove the claim let $P_{0}$ and $P_{1}$ be the centers of the squares 1 and -1 , and $Q_{0}, Q_{1}$ the centers of the squares $i$ and $-i$. Then $P_{0}$ and $P_{1}$ are fixed by $\sigma$ and $\sigma\left(Q_{0}\right)=Q_{1}$, while $-\sigma$ fixes $Q_{0}$ and $Q_{1}$ and interchanges $P_{0}$ and $P_{1}$. Moreover $P_{0}, Q_{0}, P_{1}, Q_{1}$ are (in this order) an orbit under $i$. Denoting $D$ and $D^{\prime}$ the classes in Jac $W_{\lambda}$ of the divisors $P_{1}-P_{0}$ and $Q_{0}-Q_{1}$, respectively, we find $\beta(D)=(P, 0)$ and $\beta\left(D^{\prime}\right)=(0, P)$ where $P=\varphi\left(P_{1}\right)-\varphi\left(P_{0}\right) \neq 0$ on $E$. Note that in fact $\psi\left(Q_{0}\right)-\psi\left(Q_{1}\right)=\psi \circ i\left(P_{0}\right)-\psi \circ i\left(P_{1}\right)=\varphi\left(-P_{0}\right)-\varphi\left(-P_{1}\right)=$ $\varphi\left(P_{1}\right)-\varphi\left(P_{0}\right)=P$.

Since the image of $\beta$ must be a connected subgroup, to prove surjectivity of $\beta$ it is enough to show that there is no onedimensional connected subgroup of $E \times E$ containing both $(P, 0)$ and $(0, P)$ (for some $P \neq 0)$. But all such subgroups of $E \times E$ are of the form $V(a, b)=\{(x, y) \in E \times E: a x+b y=0\}$ for some $a, b \in \mathbb{Z}[i]$; moreover $V(a, b)$ is connected if and only if $a$ and $b$ are relatively prime. Now the hypothesis $(P, 0) \in V(a, b)$ implies $a \cdot P=0$, while $(0, P) \in V(a, b)$ implies $b \cdot P=0$. Together with $\operatorname{gcd}(a, b)=1$ this gives the contradiction $P=0$.

\subsection{The cusp of $C(W)$}

Since $C(W)$ is isomorphic to $\mathbb{A}^{1}$, its closure in $\bar{M}_{3}$ contains a unique cusp $W_{\infty}$.

Proposition 8. The stable curve corresponding to $W_{\infty}$ consists of two irreducible components, both isomorphic to $E_{-1}$, that intersect transversally in two points.

Proof. $W_{\infty}$ can be obtained by contracting the (dotted) central lines of the horizontal cylinders of the origami as indicated in Figure 4.

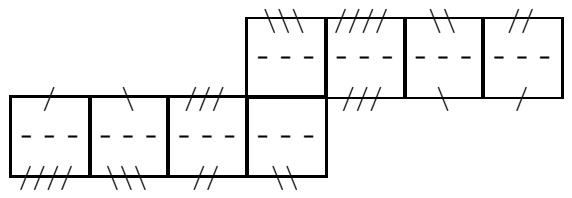

Figure 4

Clearly we obtain two irreducible components, that intersect in two points and thus both are nonsingular of genus 1 .

From Proposition 7 we know that the Jacobian of $W_{\lambda}$ contains a subvariety isogenous to $E_{-1} \times E_{-1}$, independently of $\lambda$. Therefore the Jacobian of $W_{\infty}$ must also contain such a 
subvariety. From this we deduce that the irreducible components of $W_{\infty}$ are isogenous to $E_{-1}$. Moreover we find from the picture that the automorphism $c$ of $W$ has two fixed points on each component of $W_{\infty}$ and in particular induces an automorphism on each component. As in the proof of Proposition 6 we conclude that the components are isomorphic to $E_{-1}$.

Any stable curve of genus 3 that consists of two elliptic curves intersecting in two points is hyperelliptic (i.e. in the closure of the hyperelliptic locus in $M_{3}$ ), see [HM] ex. 3.162 on p. 185]. In the case of $W_{\infty}$ the hyperelliptic involution $h$ can easily be described explicitly on the origami: $h$ acts on the "upper" horizontal cylinder like $\rho$ and on the "lower" one like $-\rho$; this means that $h$ fixes the centers of all horizontal edges and acts by rotation by $\pi$ on each of the squares around these points. Note that $h$ is not an automorphism of $W$ because the actions of $\rho$ and $-\rho$ on the two horizontal lines separating the cylinders do not agree. But on each component of $W_{\infty}, h$ acts as the multiplication by -1 , and it interchanges the two intersection points. Thus the quotient of $W_{\infty}$ by $h$ consists of two projective lines meeting transversally in one point. Together with the four punctures of $W$, two in each horizontal cylinder, this is a stable 4-marked curve of genus 0 .

It is also possible, but a bit harder, to determine $W_{\infty}$ out of the explicit formula for the family that we obtained in Proposition [5] The fibre over 0 ( or 1 or $\infty$ ) in this family is the plane curve with equation $y^{2}=x^{2}(x-1)$, which is irreducible with a tacnode at the origin; in particular it is not a stable curve. The stable reduction theorem [HM] Prop. 3.47] guarantees that there is a unique stable curve that, after passing to a branched covering of the base, fits as special fibre into this family. To find it we first have to desingularize the total space of the family. This requires three consecutive blow-ups in the singular point. After that the special fibre contains a nonreduced rational irreducible component (of multiplicity 2 ). Taking a double covering which is ramified precisely over the reduced components of the special fibre transforms the double line into a reduced elliptic curve $E$. It turns out that the other components of the special fibre are a second elliptic curve $E^{\prime}$ which intersects $E$ in two points, and two projective lines $L_{1}, L_{2}$ each of which intersects $E^{\prime}$ in one point. So far the special fibre is only semistable, but the total space is nonsingular. Finally we have to contract $L_{1}$ and $L_{2}$ to obtain a stable special fibre, although this unavoidably leads to two singular points in the total space. The details of this construction are worked out in $[\mathrm{B}$.

\section{Other maps to elliptic curves}

In this section we calculate the action of the elements of $G=\operatorname{Aut}(W)$ on $W^{1}$. We obtain explicit formulas for the quotient maps of $W$ with respect to some of the automorphisms in $G$. In particular we can determine their ramification points. This will enable us to show in Section 3 that the quaternion origami curve $C(W)$ intersects infinitely many other origami curves.

\subsection{Formulas for the automorphisms}

In Section 1.3 we described the automorphisms of $W$ by their action on the origami. Using the equation of Proposition 5 for the curve $W_{\lambda}$ we translate this into formulas which are summarized in Proposition 9

Recall that the central automorphism $c$ of $G$ has order 4 and descends to an involution of $E_{\lambda}=W_{\lambda} /\{ \pm 1\}$, with the quotient map given in Proposition 5 Thus $c$ acts in homogenous coordinates by

$$
c:(X: Y: Z) \mapsto(X: i Y: Z) .
$$

Here we made a choice between $c$ and $-c$ which corresponds to the choice between the given map and the map $(X: Y: Z) \mapsto(X:-i Y: Z)$.

\footnotetext{
${ }^{1}$ Such formulas can also be found in $[\mathrm{KK}$ ] and $\mathrm{Gi}$
} 
The four vertices of the origami squares are the four fixed points of $c$ :

$$
\begin{aligned}
P_{0} & =(0: 0: 1), & & P_{1}=(1: 0: 1), \\
P_{\infty} & =(1: 0: 0), & & P_{\lambda}=(\lambda: 0: 1) .
\end{aligned}
$$

Their images on $E_{\lambda}$ are the points $Q_{0}=P_{0}, Q_{1}=P_{1}, Q_{\infty}=(0: 1: 0)$ and $Q_{\lambda}=P_{\lambda}$. Once $Q_{\infty}$ is fixed as the origin for the group law on $E_{\lambda}$, these are the points of order 2 , see Proposition 3 We choose the translation structure on $E_{\lambda}$ such that the horizontal and vertical neighbours of $Q_{0}$ are $Q_{1}$ and $Q_{\infty}$, respectively. Lifting the structure to $W_{\lambda}$ the vertices of the squares are labeled as follows:

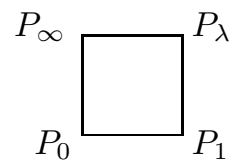

That this choice can be made in a consistent way for the whole family reflects the fact that the family $\mathcal{E}:=\left\{E_{\lambda}: \lambda \in \dddot{\mathbb{P}}\right\}$ carries a natural (even universal) level 2 structure.

By our normalization $\sigma$ interchanges $P_{0}$ with $P_{\lambda}$ and $P_{1}$ with $P_{\infty}$, see Section 1.3 In the same way, $\rho$ interchanges $P_{0}$ with $P_{1}$ and $P_{\infty}$ with $P_{\lambda}$, and $\tau$ interchanges $P_{0}$ with $P_{\infty}$ and $P_{1}$ with $P_{\lambda}$.

Any automorphism in $G$ has to permute these four points, and no element of $G-\langle c\rangle$ fixes any of them. Thus $G$ acts through the normal subgroup $V_{4}$ of $S_{4}$.

If we represent the automorphisms of $W_{\lambda}$ by matrices in $\mathrm{PGL}_{3}(\mathbb{C})$ with respect to the homogeneous coordinates $X, Y, Z$, then $c$ is given by

$$
\tilde{c}:=\left(\begin{array}{ccc}
1 & 0 & 0 \\
0 & i & 0 \\
0 & 0 & 1
\end{array}\right) .
$$

Since each $g \in G$ commutes with $c$, it is represented by a matrix $\tilde{g}$ such that $\tilde{g} \tilde{c} \tilde{g}^{-1} \tilde{c}^{-1}=\mu \cdot I_{3}$ for some $\mu \in \mathbb{C}^{\times}$and the unit matrix $I_{3}$. The determinant of a commutator is 1 ; this implies $\mu^{3}=1$. Let $x$ be an eigenvector of $\tilde{c}$ for the eigenvalue $i$. Then $\tilde{g}(x)$ is an eigenvector of $\tilde{c}$ for the eigenvalue $i \mu^{-1}$, because $\tilde{c}(\tilde{g}(x))=\mu^{-1} \tilde{g} \tilde{c}(x)=\mu^{-1} \tilde{g}(i x)$. But the only eigenvalues of $\tilde{c}$ are $i$ and 1 , hence $\mu$ must be 1 and so $\tilde{g}$ commutes with $\tilde{c}$. Therefore there is a basis of common eigenvectors of $\tilde{c}$ and $\tilde{g}$. This implies that $\tilde{g}$ has the form

$$
\left(\begin{array}{lll}
a & 0 & b \\
0 & \zeta & 0 \\
c & 0 & d
\end{array}\right) \quad \text { for some } \zeta, a, b, c, d \in \mathbb{C} .
$$

Now let $\tilde{g}$ be a matrix representing one of the automorphisms $\sigma c^{\nu}, \nu=0,1,2,3$, i. e. $\pm \sigma$ or $\pm k$ (recall that $c=k \sigma$ and $c^{2}=-1$ ). From the fact that they all interchange $P_{0}$ with $P_{\lambda}$ and $P_{1}$ with $P_{\infty}$, it is immediate to deduce the conditions $b=-\lambda a, c=a, d=-a$. The corresponding automorphism $\alpha_{\zeta}$ of $\mathbb{P}^{2}$ is therefore:

$$
\alpha_{\zeta}(X: Y: Z)=(X-\lambda Z: \zeta Y: X-Z) .
$$

Finally, $\zeta$ is determined by the fact that $\alpha_{\zeta}$ has to map $W_{\lambda}$ onto itself:

$$
\zeta^{4} Y^{4}=(X-\lambda Z)(X-Z) Z(1-\lambda) X(1-\lambda)=(1-\lambda)^{2} X Z(X-Z)(X-\lambda Z)
$$

This gives the condition

$$
\zeta^{4}=(1-\lambda)^{2} .
$$

The four possible values of $\zeta$ correspond to the four elements $\pm \sigma, \pm k$. To find out which values correspond to the elements of order 2 (i.e. $\pm \sigma$ ) we look at the square of $\alpha_{\zeta}$ :

$$
\alpha_{\zeta}^{2}(X: Y: Z)=\left(X(1-\lambda): \zeta^{2} Y: Z(1-\lambda)\right)
$$

Thus $\alpha_{\zeta}$ has order 2 if and only if $\zeta^{2}=1-\lambda$.

Carrying out the analogous calculations for the other elements of $G$ we obtain 
Proposition 9. For any $\lambda \in \dddot{\mathbb{P}}$ the 16 elements of $G$ act on $W_{\lambda}$ through the following projective automorphisms

$$
\begin{aligned}
\iota_{\nu}(X: Y: Z) & =\left(X: i^{\nu} Y: Z\right), \quad \nu=0, \ldots, 3 \\
\alpha_{\zeta}(X: Y: Z) & =(X-\lambda Z: \zeta Y: X-Z), \quad \zeta^{4}=(1-\lambda)^{2} \\
\beta_{\xi}(X: Y: Z) & =\left(X-Z: \xi Y: \frac{1}{\lambda} X-Z\right), \quad \xi^{4}=\left(1-\frac{1}{\lambda}\right)^{2} \\
\gamma_{\eta}(X: Y: Z) & =(\lambda Z: \eta Y: X), \quad \eta^{4}=\lambda^{2}
\end{aligned}
$$

The correspondence with the elements of $G$ is:

$$
\begin{aligned}
& 1=\iota_{0}, \quad-1=\iota_{2} \quad \text { and } \quad \pm c=\iota_{\nu} \text { for } \quad \nu=1,3 \\
& \pm \sigma=\alpha_{\zeta} \text { for } \zeta^{2}=1-\lambda \quad \text { and } \quad \pm k=\alpha_{\zeta} \text { for } \zeta^{2}=\lambda-1 \\
& \pm \rho=\beta_{\xi} \text { for } \xi^{2}=1-\frac{1}{\lambda} \quad \text { and } \quad \pm i=\beta_{x} i \text { for } \xi^{2}=\frac{1}{\lambda}-1 \\
& \pm \tau=\gamma_{\eta} \text { for } \eta^{2}=\lambda \text { and } \quad \pm j=\gamma_{\eta} \text { for } \eta^{2}=-\lambda
\end{aligned}
$$

\subsection{Quotient by $\sigma$}

In Proposition [6] we saw that the quotient map with respect to the automorphism $\sigma$ is a map of degree 2 from $W_{\lambda}$ to $E_{-1}$. In this section we obtain an explicit formula for this map $\kappa$ stated in Proposition 10.

Proposition 9 gives us two possibilities for $\sigma$. We fix a square $\operatorname{root} \zeta$ of $1-\lambda$ and call $\sigma_{\lambda}$ the automorphism given by

$$
\sigma_{\lambda}(X: Y: Z)=(X-\lambda Z: \zeta Y: X-Z) .
$$

The other possible choice is $-\zeta$; it describes the automorphism $(-\sigma)_{\lambda}$.

$\sigma_{\lambda}$ leaves the affine plane $Y \neq 0$ invariant, and it acts on the affine coordinates $x=\frac{X}{Y}$ and $z=\frac{Z}{Y}$ by

$$
\sigma_{\lambda}(x, z)=\left(\frac{1}{\zeta}(x-\lambda z), \frac{1}{\zeta}(x-z)\right) .
$$

The algebra of polynomials in $x$ and $z$ that are invariant under $\sigma_{\lambda}$ is generated by

$$
\begin{aligned}
p_{2}^{\prime} & :=x+\sigma_{\lambda}(x)=x\left(1+\frac{1}{\zeta}\right)-\frac{\lambda}{\zeta} \cdot z \\
\text { and } \quad p_{3}^{\prime} & :=x \cdot \sigma_{\lambda}(x)=\frac{1}{\zeta} x(x-\lambda z) .
\end{aligned}
$$

We shall work with

$$
\begin{aligned}
p_{2} & :=\frac{\zeta}{1+\zeta} \cdot p_{2}^{\prime}=x-(1-\zeta) z \\
\text { and } \quad p_{3} & :=\zeta \cdot p_{3}^{\prime}=x(x-\lambda z) .
\end{aligned}
$$

The intersection of $W_{\lambda}$ with the affine plane $Y \neq 0$ is the affine curve with the equation

$$
1=x z(x-z)(x-\lambda z) .
$$

This polynomial is invariant under $\sigma_{\lambda}$, hence can be written as a polynomial in $p_{2}$ and $p_{3}$ : Observe that

$$
\begin{aligned}
p_{2}^{2}-p_{3} & =x^{2}+(1-\zeta)^{2} z^{2}-2 x z(1-\zeta)-x^{2}+\lambda x z \\
& =(1-\zeta)^{2} z^{2}-x z\left(2-2 \zeta-1+\zeta^{2}\right)=-(1-\zeta)^{2} z(x-z) .
\end{aligned}
$$

Thus the affine equation for $W_{\lambda}$ can be rewritten as

$$
1=p_{3}\left(p_{2}^{2}-p_{3}\right) \cdot\left(-(1-\zeta)^{2}\right)^{-1} .
$$


Considering $p_{2}$ and $p_{3}$ as the affine coordinates for $\mathbb{A}^{2} /\left\langle\sigma_{\lambda}\right\rangle$, (33) is also the affine equation for $W_{\lambda} /<\sigma_{\lambda}>$.

To bring this cubic equation into Weierstrass form we first pass to the projective closure by homogenizing (3):

$$
p_{2}^{2} p_{3}=-(1-\zeta)^{2} w^{3}+w p_{3}^{2} .
$$

Choose a square root $\omega$ of $1-\zeta$ and rewrite this equation in the coordinates $\tilde{p}_{2}=\omega p_{2}$, $\tilde{p}_{3}=p_{3}, \tilde{w}:=-(1-\zeta) w:$

$$
\tilde{p}_{2}^{2} \tilde{p}_{3}=(1-\zeta) p_{2}^{2} p_{3}=-(1-\zeta)^{3} w^{3}+(1-\zeta) w p_{3}^{2}=\tilde{w}^{3}-\tilde{w} \tilde{p}_{3}^{2} .
$$

This is the standard Weierstrass form of $E_{-1}$. Putting everything together we get

Proposition 10. The quotient map $\kappa=\kappa_{\sigma_{\lambda}}: W_{\lambda} \rightarrow W_{\lambda} /\left\langle\sigma_{\lambda}\right\rangle=E_{-1}$ is given by

$$
\kappa(X: Y: Z)=\left(-(1-\zeta) Y^{2}: \omega Y(X-(1-\zeta) Z): X(X-\lambda Z)\right)
$$

for $(X: Y: Z) \notin\left\{P_{0}, P_{\lambda}\right\}$ and $\kappa\left(P_{0}\right)=\kappa\left(P_{\lambda}\right)=(0: 1: 0)$.

$\kappa$ is determined by $\lambda$ uniquely up to the sign of $\zeta=\sqrt{1-\lambda}$ (which determines the choice between $\sigma_{\lambda}$ and $(-\sigma)_{\lambda}$ ), and the sign of $\omega=\sqrt{1-\zeta}$ (a change of which corresponds to a composition with the automorphism $[-1]$ on $\left.E_{-1}\right)$.

Proof. Considered on the appropriate affine parts of $\mathbb{P}^{2}$, the affine quotient map $(x, y) \mapsto$ $\left(p_{2}, p_{3}\right)$ is given by $\left(\frac{X}{Y}: 1: \frac{Z}{Y}\right) \mapsto\left(1: \frac{p_{2}}{w}: \frac{p_{3}}{w}\right)$. The change of coordinates from $w, p_{2}, p_{3}$ to $\tilde{w}, \tilde{p}_{2}, \tilde{p}_{3}$ transforms this into $\left(1: \frac{\omega p_{2}}{-(1-\zeta) w}: \frac{p_{3}}{-(1-\zeta) w}\right)$. Substituting $\frac{X}{Y}-(1-\zeta) \frac{Z}{Y}$ for $\frac{p_{2}}{w}$ and $\frac{X}{Y}\left(\frac{X}{Y}-\lambda \frac{Z}{Y}\right)$ for $\frac{p_{3}}{w}$ gives the formula on $W_{\lambda} \cap\{Y \neq 0\}$. To determine the image of $\kappa$ in the remaining points $P_{0}$ and $P_{\lambda}$, replace the third component $X(X-\lambda Z)$ by $\frac{Y^{2}}{X(X-Z)}$ and multiply all components by $\frac{X(X-Z)}{Y}$. Thus on the open set $\{X \neq 0, X \neq Z, Y \neq 0\}$, we also have $\kappa(X: Y: Z)=\left(-(1-\zeta) Y Z(X-Z): \omega(X-(1-\zeta) Z) Z(X-Z): Y^{3}\right)$. This formula gives us $\kappa\left(P_{0}\right)$ and $\kappa\left(P_{\lambda}\right)$.

\subsection{The other involutions}

To determine the quotient maps $W_{\lambda} \rightarrow E_{-1}$ with respect to the automorphisms $\rho$ and $\tau$ we proceed as in Section 2.2

Out of the two possibilities for $\rho$ in Proposition 9 we pick one by fixing a square root $\xi$ of $1-\frac{1}{\lambda}$ and denoting $\rho_{\lambda}$ the automorphism of $W_{\lambda}$ which in affine coordinates $x=\frac{X}{Y}, z=\frac{Z}{Y}$ is given by

$$
\rho_{\lambda}(x, z)=\left(\frac{1}{\xi}(x-z), \frac{1}{\xi}\left(\frac{1}{\lambda} x-z\right)\right) .
$$

As in the case of $\sigma$, the other square root $-\xi$ then describes $(-\rho)_{\lambda}$.

This time, the invariants are generated by

$$
p_{2}=x-\frac{1}{\xi+1} z \text { and } p_{3}=x(x-z) .
$$

In these invariants, the right hand side of the equation defining $W_{\lambda}$ is

$$
x z(x-z)(x-\lambda z)=-p_{3}\left(p_{2}^{2}-p_{3}\right) \cdot(1+\xi)^{2} \lambda,
$$

which leads to the affine equation

$$
p_{2}^{2} p_{3}-p_{3}^{2}=-\frac{1}{\lambda(1+\xi)^{2}}
$$

for $\left.W_{\lambda} /<\rho_{\lambda}\right\rangle$. As before we homogenize this equation with a variable $w$ and take a homogeneous change of coordinates to transform this equation into Weierstrass form:

$$
\tilde{p}_{2}=\omega_{\rho} p_{2}, \tilde{w}=-\omega_{\rho}^{2} w, \tilde{p}_{3}=p_{3}
$$


with $\omega_{\rho}^{4}=\frac{1}{\lambda(1+\xi)^{2}}$. This gives us the quotient map $\kappa_{\rho}=\kappa_{\rho_{\lambda}}$ stated below in Proposition 11 Finally $\tau$ acts in affine coordinates by

$$
\tau_{\lambda}(x, z)=\left(\frac{\lambda}{\eta} z, \frac{1}{\eta} x\right)
$$

where we fix a square root $\eta$ of $\lambda$ and thus distinguish $\tau_{\lambda}$ from $(-\tau)_{\lambda}$.

The same reasoning as above gives the quotient map $\kappa_{\tau}: W_{\lambda} \rightarrow W_{\lambda} /\left\langle\tau_{\lambda}\right\rangle=E_{-1}$ in the following proposition.

Proposition 11. The quotient maps $\kappa_{\rho}=\kappa_{\rho_{\lambda}}: W_{\lambda} \rightarrow W_{\lambda} /\left\langle\rho_{\lambda}\right\rangle=E_{-1}$ and $\kappa_{\tau}: W_{\lambda} \rightarrow$ $\left.W_{\lambda} /<\tau_{\lambda}\right\rangle=E_{-1}$ are given as follows:

$$
\begin{aligned}
& \kappa_{\rho}(X: Y: Z)=\left(-\omega_{\rho}^{2} Y^{2}: \omega_{\rho} Y\left(X-\frac{1}{1+\xi} Z\right): X(X-Z)\right) \\
& \quad \text { for }(X: Y: Z) \notin\left\{P_{0}, P_{1}\right\} \text { and } \kappa_{\rho}\left(P_{0}\right)=\kappa_{\rho}\left(P_{1}\right)=(0: 1: 0) . \\
& \kappa_{\tau}(X: Y: Z)=\left(\frac{i}{1+\eta} Y^{2}: \omega_{\tau} Y(X+\eta Z): X Z\right) \\
& \quad \text { for }(X: Y: Z) \notin\left\{P_{0}, P_{\infty}\right\} \text { and } \kappa_{\tau}\left(P_{0}\right)=\kappa_{\tau}\left(P_{\infty}\right)=(0: 1: 0) . \\
& \quad \text { Here } \omega_{\tau} \text { is a square root of }\left(\frac{i}{1+\eta}\right)^{3} .
\end{aligned}
$$

$\kappa_{\rho}$ is determined by $\lambda$ up to the sign of $\xi$ (which gives the choice between $\rho_{\lambda}$ and $(-\rho)_{\lambda}$ ), and up to multiplying $\omega_{\rho}$ by a power of $i$ (which results in composing $\kappa_{\rho}$ with a power of the automorphism $\bar{c}$ of $\left.E_{-1}\right) . \kappa_{-\rho}$ is obtained from $\kappa_{\rho}$ by replacing $\xi$ by $-\xi$. Similarly $\kappa_{\tau}$ is determined up tp the signs of $\eta$ and $\omega_{\tau}$.

Next we shall show that all these quotient maps are closely related to each other. First recall that $W_{\lambda}$ is isomorphic to $W_{\lambda^{\prime}}$ iff $\lambda^{\prime} \in\left\{\lambda, \frac{1}{\lambda}, 1-\lambda, \frac{1}{1-\lambda}, 1-\frac{1}{\lambda}, \frac{\lambda}{\lambda-1}\right\}$ (see proof of Proposition 5). Explicitly,

$$
\varphi_{1}(X: Y: Z)=\left(Z: \frac{1}{\sqrt[4]{\lambda}} Y: X\right)
$$

induces an isomorphism $\varphi_{1}: W_{\lambda} \rightarrow W_{\frac{1}{\lambda}}$, and

$$
\varphi_{2}(X: Y: Z)=\left(Z-X: \zeta_{8} Y: Z\right)
$$

induces an isomorphism $\varphi_{2}: W_{\lambda} \rightarrow W_{1-\lambda}$; here $\zeta_{8}$ is a primitive eighth root of 1 . The other isomorphisms can be obtained from these two by composition, since $s_{1}(\lambda)=\frac{1}{\lambda}$ and $s_{2}(\lambda)=1-\lambda$ generate the above group of six transformations.

The following relations can be checked by straightforward calculation:

\section{Proposition 12.}

$$
\begin{array}{ll}
\varphi_{1}^{-1} \circ \sigma_{\frac{1}{\lambda}} \circ \varphi_{1}=(-\rho)_{\lambda} & \varphi_{2}^{-1} \circ \sigma_{1-\lambda} \circ \varphi_{2}=(-\tau)_{\lambda} \\
\varphi_{1}^{-1} \circ \rho_{\frac{1}{\lambda}} \circ \varphi_{1}=(-\sigma)_{\lambda} & \varphi_{2}^{-1} \circ \rho_{1-\lambda} \circ \varphi_{2}=\rho_{\lambda} \\
\varphi_{1}^{-1} \circ \tau_{\frac{1}{\lambda}} \circ \varphi_{1}=\tau_{\lambda} & \varphi_{2}^{-1} \circ \tau_{1-\lambda} \circ \varphi_{2}=(-\sigma)_{\lambda} .
\end{array}
$$

In these equations the sign of the automorphism on the right hand side depends on the choice of the square roots $\zeta(\lambda), \xi(\lambda)$ and $\eta(\lambda)$. E. g. the first equation is correct in this form if we choose $\xi(\lambda)=\zeta\left(\frac{1}{\lambda}\right)$.

Corollary 13. There is an isomorphism $\bar{\varphi}_{1}: E_{-1} \rightarrow E_{-1}$ such that $\kappa_{\sigma}^{\left(\frac{1}{\lambda}\right)} \circ \varphi_{1}=\bar{\varphi}_{1} \circ \kappa_{-\rho}^{(\lambda)}$, i. e. $\bar{\varphi}_{1}$ makes the following diagram commutative:

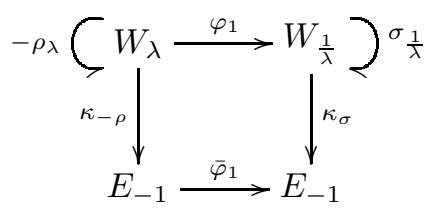


Explicitly, $\bar{\varphi}_{1}$ is the translation by $(0: 0: 1)$.

Such an isomorphism exists in an analogous way for each of the isomorphisms $W_{\lambda} \rightarrow W_{\lambda^{\prime}}$, $\lambda^{\prime} \in\left\{\lambda, \frac{1}{\lambda}, 1-\lambda, \frac{1}{1-\lambda}, 1-\frac{1}{\lambda}, \frac{\lambda}{\lambda-1}\right\}$.

\subsection{Ramification points}

In this section we calculate the ramification points and the critical points of the two-sheeted coverings $W_{\lambda} \rightarrow E_{-1}$, i. e. the fixed points of the involutions $\pm \sigma, \pm \rho$ and $\pm \tau$ on $W_{\lambda}$ and their images on $E_{-1}$ under $\kappa_{\sigma}$ (resp. $\kappa_{-\sigma}, \kappa_{ \pm \rho}$ and $\kappa_{ \pm \tau}$ ).

Proposition 14. For any $\alpha \in\{ \pm \sigma, \pm \rho, \pm \tau\}$ the fixed points of $\alpha$ on $W_{\lambda}$ are

$$
P_{\alpha}^{\nu}:=\left(X_{\alpha}: i^{\nu}: Z_{\alpha}\right), \nu=0,1,2,3,
$$

where $X_{\alpha}$ and $Z_{\alpha}$ are defined as follows using the notation $\zeta, \xi$ and $\eta$ as in the previous section:

$$
\begin{aligned}
X_{\sigma} & =(1+\zeta) Z_{\sigma}, & Z_{\sigma} & =\frac{1}{\sqrt{\zeta(1+\zeta)}}, \\
X_{\rho} & =\sqrt[4]{\frac{1+\xi}{\xi^{2}(1-\xi)}}, & Z_{\rho} & =(1-\xi) X_{\rho} \\
X_{\tau} & =\eta Z_{\tau}, & Z_{\tau} & =\frac{1}{\sqrt{i \eta(\eta-1)}} .
\end{aligned}
$$

As in section 2.3 the fixed points of $-\sigma($ resp. $-\rho,-\tau)$ are obtained from these formulas by replacing $\zeta$ (resp. $\xi, \eta)$ by its negative.

A different choice of a root in these expressions results in a permutation of the four points $P_{\alpha}^{\nu}, \nu=0,1,2,3$.

Proof. Recall that $W_{\lambda} \cap\{Y=0\}=\left\{P_{0}, P_{1}, P_{\infty}, P_{\lambda}\right\}$, and none of these points is fixed by any of the six involutions. So we may assume all fixed points in the form $(X: 1: Z)$. If such a point is fixed by $\sigma$, we see from Proposition 9 that $Z=\frac{1}{\zeta}(X-Z)$ or equivalently $X=Z(1+\zeta)$. Inserting this into the equation for $W_{\lambda}$ we find

$$
1=Z^{4}(1+\zeta) \zeta(1+\zeta-\lambda)=Z^{4} \zeta^{2}(1+\zeta)^{2},
$$

which gives $Z_{\sigma}$. The calculations for $\rho$ and $\tau$ are similar.

Remark that $P_{\alpha}^{0}, P_{\alpha}^{1}, P_{\alpha}^{2}, P_{\alpha}^{3}$ form an orbit under the automorphism $c$. This can also be seen from the description of the automorphisms in Section 1.3

The critical points of $\kappa_{\alpha}$ are the images of the $P_{\alpha}^{\nu}$, e.g.:

$$
\begin{aligned}
\kappa_{\sigma}\left(P_{\sigma}^{\nu}\right) & =\left((-1)^{\nu+1}(1-\zeta): i^{\nu} \sqrt{1-\zeta} \frac{2 \zeta}{\sqrt{\zeta(1+\zeta)}}:(1+\zeta) \frac{1+\zeta-\lambda}{\zeta(1+\zeta)}\right) \\
& =\left((-1)^{\nu+1}(1-\zeta): i^{\nu} \sqrt{\frac{1-\zeta}{1+\zeta}} 2 \sqrt{\zeta}: 1+\zeta\right) \\
& =\left((-1)^{\nu+1} \lambda: 2 i^{\nu} \sqrt{\zeta \lambda}:(1+\zeta)^{2}\right)
\end{aligned}
$$

Proceeding in the same way for the other five involutions we obtain

Corollary 15. The images in $E_{-1}$ of the fixed points of $\pm \sigma, \pm \rho$ and $\pm \tau$ under $\kappa_{ \pm \sigma}, \kappa_{ \pm \rho}$ 
and $\kappa_{ \pm \tau}$ resp., are

$$
\begin{aligned}
Q_{\sigma} & =\left(-\lambda: 2 \sqrt{\zeta \lambda}:(1+\zeta)^{2}\right) \\
Q_{-\sigma} & =\left(\lambda: 2 \sqrt{\zeta \lambda}:(1-\zeta)^{2}\right) \\
Q_{\rho} & =(\xi-1: 2 \eta(1-\xi) \sqrt{\xi}: 1+\xi) \\
Q_{-\rho} & =(\xi+1: 2 \eta(1+\xi) \sqrt{\xi}: 1-\xi) \\
Q_{\tau} & =\left(1-\eta: 2 \sqrt{\eta} \sqrt{\frac{\eta-1}{\eta+1}}: \eta+1\right) \\
Q_{-\tau} & =\left(1+\eta: 2 \sqrt{\eta} \sqrt{\frac{\eta+1}{\eta-1}}: \eta-1\right)
\end{aligned}
$$

and their orbits under the automorphism $\bar{c}$.

\section{$3 \quad$ Intersecting origami curves}

Now, finally we show (in 3.1) that in the moduli space $M_{3}$ the Teichmüller curve $C(W)$ of the quaternion origami intersects infinitely many other origami curves. The combinatorial description of these origamis is given in 3.2

\subsection{The main result}

Recall from Proposition 10 that we have for each $\lambda \in \dddot{\mathbb{P}}$ the degree 2 morphism $\kappa=\kappa_{\sigma_{\lambda}}$ : $W_{\lambda} \rightarrow E_{-1}$. The critical points of $\kappa$ form an orbit under the order 4 automorphism $\bar{c}$ and are given explicitly in Corollary 15 These are the main ingredients for the following theorem.

Theorem 1. The origami curve $C(W)$ intersects infinitely many other origami curves.

Proof. The idea of the proof is to compose $\kappa_{\sigma}$ (or one of the other morphisms $W_{\lambda} \rightarrow E_{-1}$ ) with a morphism $\varphi: E_{-1} \rightarrow E^{\prime}$ to an elliptic curve $E^{\prime}$ that maps the four ramification points $Q_{\sigma}^{\nu}=\bar{c}^{\nu}\left(Q_{\sigma}\right), \nu=0,1,2,3$ to the same point. Then $\varphi \circ \kappa_{\sigma}$ is an origami.

Claim. Such a morphism exists if and only if $Q_{\sigma}:=Q_{\sigma}^{0}$ is a torsion point on $E_{-1}$ (with the usual group structure, i. e. with $0:=(0: 1: 0)$ as origin $)$.

Proof of the claim. By composing with an isogeny $E^{\prime} \rightarrow E_{-1}$ if necessary we may assume that $\varphi$ is the product of a translation with multiplication by $n$ on $E_{-1}$ (for some $n \geq 2$ ). Hence $\varphi\left(Q_{\sigma}^{\nu}\right)=\varphi\left(Q_{\sigma}\right)$ for $\nu=0, \ldots, 3$ if and only if there is a point $P \in E_{-1}(\mathbb{C})$ such that $n \cdot\left(Q_{\sigma}^{\nu}-P\right)=Q^{\prime}$ with the same point $Q^{\prime}$ for $\nu=0, \ldots, 3$. On the other hand, since $Q_{\sigma}^{\nu}=\bar{c}^{\nu}\left(Q_{\sigma}\right)$, we have $n \cdot \bar{c}^{\nu}\left(Q_{\sigma}\right)=n \cdot P+Q^{\prime}$ for $\nu=0, \ldots, 3$. Since $\bar{c}$ commutes with multiplication by $n$, this implies in particular that $n \cdot Q_{\sigma}$ is a fixed point of $\bar{c}$, i. e. either 0 or $(0: 0: 1)$. In both cases $2 n \cdot Q_{\sigma}=0$. This proves the claim.

From Section 2.4 we know that

$$
\begin{aligned}
Q_{\sigma}=Q_{\sigma}(\lambda) & =\left(-\lambda: 2 \sqrt{\zeta \lambda}:(1+\zeta)^{2}\right)=\left(-\frac{\lambda}{(1+\zeta)^{2}}: 2 \frac{\sqrt{\zeta \lambda}}{(1+\zeta)^{2}}: 1\right) \\
& =\left(\frac{\zeta-1}{\zeta+1}: 2 \frac{\sqrt{\zeta \lambda}}{(1+\zeta)^{2}}: 1\right) \in E_{-1}: y^{2}=x^{3}-x
\end{aligned}
$$

As $W_{\lambda}$ runs through $C(W), \lambda$ takes on every value in $\dddot{\mathbb{P}}=\mathbb{P}^{1}(\mathbb{C})-\{0,1, \infty\}$. Since $\zeta^{2}=1-\lambda$, $\zeta$ omits only the values $0, \infty$ and \pm 1 , and the same holds for $\frac{\zeta-1}{\zeta+1}$. This shows that for every $Q \in E_{-1}$ which is not a 2 -torsion point we find $\lambda \in \dddot{\mathbb{P}}$ such that $Q_{\sigma}(\lambda)=Q$. Together with the claim this proves that for every torsion point $T$ of $E_{-1}$ of (exact) order $n \geq 3$ there is $\lambda_{T} \in \dddot{\mathbb{P}}$ such that $[n] \circ \kappa_{\sigma}: W_{\lambda_{T}} \rightarrow E_{-1}$ defines an origami $D_{T}$ which is different from $W$. By construction $C\left(D_{T}\right)$ and $C(W)$ intersect in $W_{\lambda_{T}}$. 


\subsection{Nice double coverings of the trivial origami}

In this section we describe the origamis intersecting $C(W)$.

Recall from the last section that they are given as composition of the double cover $\kappa: W_{\lambda} \rightarrow$ $E_{-1}$ and $[n]: E_{-1} \rightarrow E_{-1}$, the multiplication with $n$. The four critical points of $\kappa$ are $n$ torsion points but not 2-torsion points. They form an orbit under the automorphism $\bar{c}$ on $E_{-1}$ which has order four and fixed points 0 and $(0: 0: 1)$. Furthermore, $\bar{c}$ is induced by the automorphism $c$ on $W_{\lambda}$ and $c$ as well as $c^{2}$ have four fixed points.

Call $E^{[1]}$ the origami with the translation structure defined by the unit lattice on $E_{-1}$. Furthermore, call $E^{[n]}$, respectively $D$, the origami given as the pullback via $[n]$ on $E_{-1}$, respectively via $[n] \circ \kappa$ on $W_{\lambda}$. Then $D$ is the desired origami and $E^{[n]}$ consists of a large square of length $n$ subdivided into $n^{2}$ squares (see Figure 5) where opposite sides are glued. We will show that the properties listed in the last paragraph uniquely determine $D$.

Remark that the automorphisms $\bar{c}$ and $\bar{c}^{-1}$ are affine with derivative

$$
S:=\left(\begin{array}{cc}
0 & -1 \\
1 & 0
\end{array}\right) \text { and } S^{-1}:=\left(\begin{array}{cc}
0 & 1 \\
-1 & 0
\end{array}\right), \text { resp. }
$$

since they are the only automorphisms of order four fixing 0 . The lift $c$ is thus also affine with the same derivative.

Proposition 16. Let $P$ be a n-torsion point of $E^{[n]}$ which is not a 2-torsion point. Then, there is a uniquely determined origami $D=\left(p: X \rightarrow E^{[1]}\right)$ that has a translation covering $q: X \rightarrow E^{[n]}$ of degree 2 with $p=[n] \circ q$ such that the following properties hold:

1. There is an affine automorphism $c$ on $X$ with derivative $D(c)=S$ that descends to the rotation $\bar{c}$ on $E^{[n]}$ with derivative $S$ and center 0 . The automorphism $c$ and its square $c^{2}$ have four fixed points on $X$.

2. The critical points of $q$ are $P, Q:=\bar{c}(P), R:=\bar{c}^{2}(P)$ and $S:=\bar{c}^{3}(P)$.

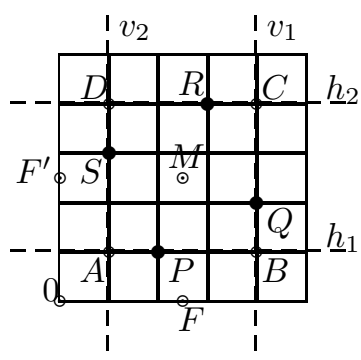

Figure 5

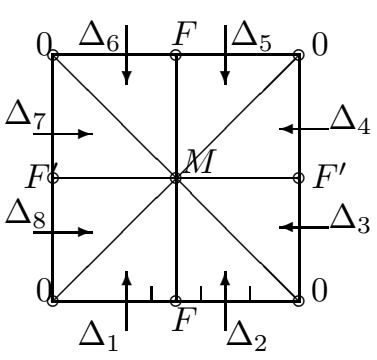

Figure 6

Proof. First we show that there is at most one origami like this. Suppose $D=\left(p: X \rightarrow E^{[1]}\right)$ is an origami with a covering $q: X \rightarrow E^{[n]}$ fulfilling the properties in the proposition. Draw - as shown in Figure [5- the horizontal lines $h_{1}$ through $P$ and $h_{2}$ through $R$ and the vertical lines $v_{1}$ through $Q$ and $v_{2}$ through $S$. Define $A:=h_{1} \cap v_{2}, B:=h_{1} \cap v_{1}, C:=h_{2} \cap v_{1}$, $D:=h_{2} \cap v_{2}$. Let $M \neq 0$ be the other fixed point of $\bar{c}$ and let $F$ and $F^{\prime}$ be the other 2-torsion points of $E^{[n]}$ on the horizontal and the vertical geodesic starting in 0 .

$E^{[n]}$ is divided by the horizontal and vertical lines through 0 and $M$ and the two diagonals into eight (euclidian) geodesic triangles $\Delta_{1}, \ldots, \Delta_{8}$ (see Figure 6). We distinguish five cases:

1. $P$ lies in the open triangle $\Delta_{1}, \Delta_{3}, \Delta_{5}$ or $\Delta_{7}$.

2. $P$ lies in the open triangle $\Delta_{2}, \Delta_{4}, \Delta_{6}$ or $\Delta_{8}$.

3. $P$ lies on one of the two diagonals.

4. $P$ lies on the horizontal or the vertical line through 0 .

5. $P$ lies on the horizontal or the vertical line through $M$. 


\section{Case 1;}

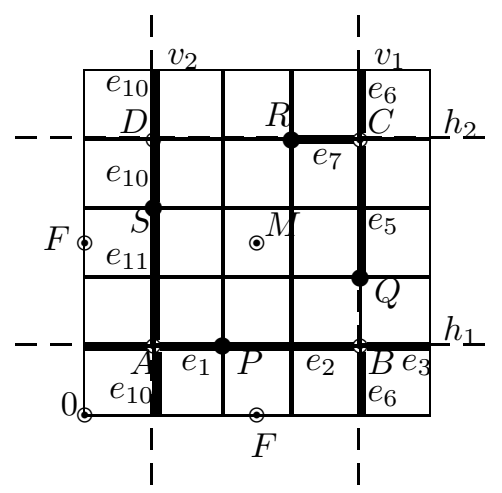

Figure 7

Suppose $P$ lies inside $\Delta_{1}$. If it lies in one of the other three triangles one can interchange the roles of $P, Q, R$ and $S$.

Since $P$ lies inside the open triangle $\Delta_{1}$, the lines $h_{1}, h_{2}, v_{1}, v_{2}$ are pairwise different, the sets $\{A, B, C, D\}$ and $\{P, Q, R, S\}$ are disjoint and we have: $P$ lies between $A$ and $B, Q$ lies between $B$ and $C, R$ lies between $D$ and $C, S$ lies between $A$ and $D^{2}$ (compare Figure 7). Define the segments $e_{1}:=A P, e_{2}:=P B, e_{3}:=B A, e_{5}:=Q C, e_{6}:=C B, e_{7}:=R C$, $e_{10}:=S A, e_{11}:=A S$ (compare Footnote 2). Removing $e_{1}, e_{2}, e_{3}, e_{5}, e_{6}, e_{7}, e_{10}, e_{11}$ from $E^{[n]}$ gives a simply connected open region $U$. Since the critical points $P, Q, R$ and $S$ are contained in the border of $U$, one can get any degree 2 cover of $E^{[n]}$ which has those as critical points by taking two copies $U_{1}$ and $U_{2}$ of $U$ and glueing the borders of $\bar{U}_{1}$ and $\bar{U}_{2}$. Now, take a glueing that gives $q: X \rightarrow E^{[n]}$. We will show that it is uniquely determined by the properties required in the proposition.

Let's label all horizontal and vertical edges $e$ of length 1 between two neighbouring $n$-torsion points of $E^{[n]}$ by a label $l(e) \in \mathbb{Z} / 2 \mathbb{Z}$ in the following way:

- $l(e)=\overline{1}$, if there is a change of the two leaves at $e$, i.e. if for a preimage $Z$ of a point in the interior of $e$, each neigbourhood of $Z$ intersects $U_{1}$ and $U_{2}$.

- $l(e)=\overline{0}$, if there is no change of the leaves at $e$, i.e. if for a preimage $Z$ of a point in the interior of $e$, there is a neigbourhood of $Z$ that lies completely inside $U_{1}$ or $U_{2}$.

By construction we have $l(e)=\overline{1} \Rightarrow e \in\left\{e_{1}, e_{2}, e_{3}, e_{5}, e_{6}, e_{7}, e_{10}, e_{11}\right\}$.

Furthermore, an $n$-torsion point on $E^{[n]}$ that is intersection of the four edges $e, e^{\prime}, e^{\prime \prime}, e^{\prime \prime \prime}$ is

$$
\begin{aligned}
& \text { - ramified } \Leftrightarrow l(e)+l\left(e^{\prime}\right)+l\left(e^{\prime \prime}\right)+l\left(e^{\prime \prime \prime}\right)=\overline{1} . \\
& \text { - unramified } \Leftrightarrow l(e)+l\left(e^{\prime}\right)+l\left(e^{\prime \prime}\right)+l\left(e^{\prime \prime \prime}\right)=\overline{0} .
\end{aligned}
$$

Hence we get by Condition 2 in the proposition:

$$
\begin{array}{lr}
l\left(e_{1}\right)+l\left(e_{2}\right)=1, \quad l\left(e_{5}\right)=1, & \\
l\left(e_{7}\right)=1, l\left(e_{10}\right)+l\left(e_{11}\right)=1 & (P, Q, R, S \text { ramified }) \\
l\left(e_{1}\right)+l\left(e_{11}\right)+l\left(e_{3}\right)+l\left(e_{10}\right)=0, & l\left(e_{2}\right)+l\left(e_{6}\right)+l\left(e_{3}\right)=0, \\
l\left(e_{5}\right)+l\left(e_{6}\right)+l\left(e_{7}\right)=0 & (A, B, C, D \text { unramified })
\end{array}
$$

By Condition 1 in the proposition the automorphism $c$ has four fixed points on $X$. Since $\bar{c}$ on $E^{[n]}$ has the two fixed points 0 and $M$, the four fixed points of $c$ are their preimages $0_{1}:=q^{-1}(\{0\}) \cap U_{1}, 0_{2}:=q^{-1}(\{0\}) \cap U_{2}, M_{1}:=q^{-1}(\{M\}) \cap U_{1}$ and $M_{2}:=q^{-1}(\{M\}) \cap U_{2}$. Obviously, they are also fixed points of $c^{2}$ and by condition 1 they are all of them. From this it follows that $l\left(e_{2}\right)=1$ : If not the vertical geodesic starting in $M_{1}$ would be a closed

\footnotetext{
${ }^{2}$ Orientation is always left to right for horizontal directions and bottom to top for vertical directions.
} 
geodesic of length $n$ and the 2-torsion point $F$ of $E^{[n]}$ on it would be a fixed point of $c^{2}$. Similarly, we get $l\left(e_{5}\right)+l\left(e_{11}\right)=1$. Hence we have

$$
l\left(e_{2}\right)=1 \quad \text { and } \quad l\left(e_{5}\right)+l\left(e_{11}\right)=1 .
$$

From the equations (4), (5) and (6) it follows that:

$$
\begin{array}{llll}
l\left(e_{1}\right)=0, & l\left(e_{2}\right)=1, & l\left(e_{3}\right)=1, & l\left(e_{5}\right)=1, \\
l\left(e_{6}\right)=0, & l\left(e_{7}\right)=1, & l\left(e_{10}\right)=1, & l\left(e_{11}\right)=0
\end{array}
$$

Hence, the origami $D$ is uniquely determined by the conditions in the proposition: the leaves are switched at the edges $e_{2}, e_{3}, e_{5}, e_{7}$ and $e_{10}$.

Conversely, this origami fulfills the condition of the proposition:

In order to see that the automorphism $\bar{c}$ of $E^{[n]}$ can be lifted to $X$ we consider the fundamental group $H$ of $E^{[n]} \backslash\{P, Q, R, S\}$. It is a free group in the generators $\alpha, \beta, \gamma_{1}, \gamma_{2}, \gamma_{3}$, where $\alpha$ and $\beta$ are the closed horizontal and vertical geodesic lines through $M$ and $\gamma_{1}, \gamma_{2}$, $\gamma_{3}$ are the positively oriented simple loops around $P, Q$ and $R$. The loop $\gamma_{4}$ around $S$ is equivalent to $\gamma_{3}^{-1} \gamma_{2}^{-1} \gamma_{1}^{-1}$.

By the definition of the glueings the monodromy $H \rightarrow \mathbb{Z} / 2 \mathbb{Z}$ is given by $g \mapsto 1$ for each generator $g$. The fundamental group $U$ of $X \backslash q^{-1}(\{P, Q, R, S\})$ is the kernel of the monodromy. Thus it is the index 2 subgroup of words of even length.

The automorphism of $H$ induced by $\bar{c}$ is given by

$$
\alpha \mapsto \beta, \quad \beta \mapsto \alpha^{-1}, \quad \gamma_{1} \mapsto \gamma_{2}, \quad \gamma_{2} \mapsto \gamma_{3}, \quad \gamma_{3} \mapsto \gamma_{4}=\gamma_{3}^{-1} \gamma_{2}^{-1} \gamma_{1}^{-1} .
$$

It preserves the parity of the length of words and thus it preserves $U$. It follows that $\bar{c}$ can be lifted to $c$ on $X$. One checks that $c$ and $c^{2}$ have the fixed point set $q^{-1}(\{0, M\})$. Thus Condition 1 is fulfilled.

Condition 2 is fulfilled since (*) holds for $P, Q, R$ and $S$ and $(* *)$ holds for $A, B, C$ and $D$. This finishes the proof for Case 1

The other four cases work in the same way.

\section{Case 2}

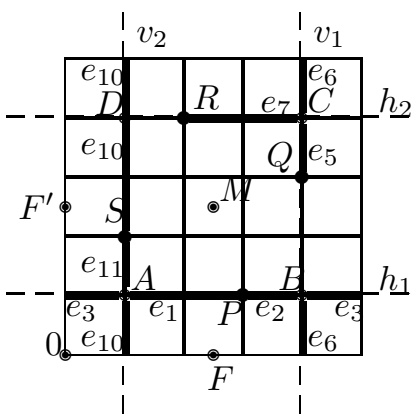

Figure 8

\section{Case 4}

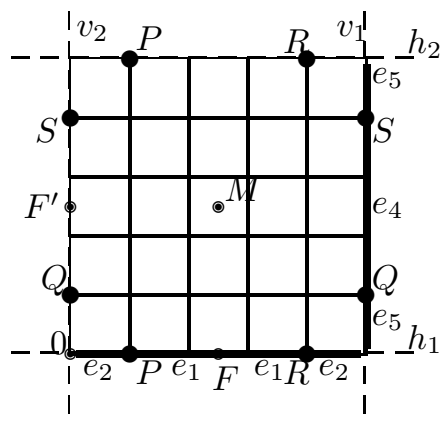

Figure 10

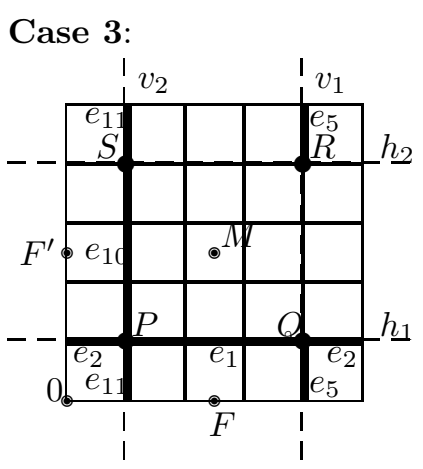

Figure 9

\section{Case 5}

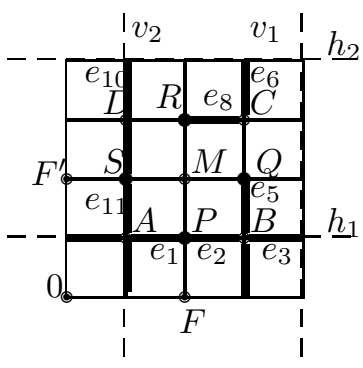

Figure 11 
Using the same arguments one obtains:

\section{Case 2]}

Removed segments: $e_{1}, e_{2}, e_{3}, e_{5}, e_{6}, e_{7}, e_{10}, e_{11}$.

$$
\begin{array}{llll}
l\left(e_{1}\right)=0, & l\left(e_{2}\right)=1, & l\left(e_{3}\right)=1, & l\left(e_{5}\right)=1 \\
l\left(e_{6}\right)=0, & l\left(e_{7}\right)=1, & l\left(e_{10}\right)=1, & l\left(e_{11}\right)=0 .
\end{array}
$$

Thus, the leaves are switched at $e_{2}, e_{3}, e_{5}, e_{7}$ and $e_{10}$.

\section{Case 3}

Removed segments: $e_{1}, e_{2}, e_{5}, e_{10}, e_{11}$.

$$
l\left(e_{1}\right)=1, \quad l\left(e_{2}\right)=1, \quad l\left(e_{5}\right)=1, \quad l\left(e_{10}\right)=1, \quad l\left(e_{11}\right)=0 .
$$

Thus, the leaves are switched at $e_{1}, e_{2}, e_{5}$ and $e_{10}$.

Case 4

Removed segments: $e_{1}, e_{2}, e_{4}, e_{5}$.

$$
l\left(e_{1}\right)=1, l\left(e_{2}\right)=0, l\left(e_{4}\right)=1, l\left(e_{5}\right)=0 .
$$

Thus, the leaves are switched at $e_{1}$ and $e_{4}$.

\section{Case [5]}

Removed segments: $e_{1}, e_{2}, e_{3}, e_{5}, e_{6}, e_{8}, e_{10}, e_{11}$.

$$
\begin{array}{llll}
l\left(e_{1}\right)=0, & l\left(e_{2}\right)=1, & l\left(e_{3}\right)=1, & l\left(e_{5}\right)=1 \\
l\left(e_{6}\right)=1, & l\left(e_{8}\right)=1, & l\left(e_{10}\right)=0, & l\left(e_{11}\right)=1 .
\end{array}
$$

Thus, the leaves are switched at $e_{2}, e_{3}, e_{5}, e_{6}, e_{8}$ and $e_{11}$.

By the same arguments as in the first case the origamis defined by these glueings fulfill the conditions in the proposition and are uniquely determined.

\section{References}

[B] Bauer, O.: Stabile Reduktion und Origamis. Diplomarbeit, Karlsruhe 2005.

[EG] Earle, C.J., Gardiner,F.P.: Teichmüller disks and Veech's F-structures. American Mathematical Society. Contemporary Mathematics 201, 165-189 (1997).

[Gi] Girard, M.: Géométrie du groupe des points de Weierstrass d'une quartique lisse. Journal of Number Theory 94, 103-135 (2002).

[Gu1] Guàrdia, J.: Explicit geometry on a family of curves of genus 3. J. London Math. Soc. (2) 64, 299-310 (2001).

[Gu2] Guàrdia, J.: A family of arithmetic surfaces of genus 3. Pacific Journal of Mathematics 212, 71-91 (2003).

[GJ] Gutkin, E., Judge, C.: Affine mappings of translation surfaces: Geometry and arithmetic. Duke Mathematical Journal 103 No. 2, 191-213 (2000).

[HaSe] Hall, M., Senior, P.: The groups of order $2^{n}(n \leq 6)$. Macmillan, New York 1964.

[HM] Harris, J., Morrison, I.: Moduli of Curves. Springer Grad. Texts in Math. 187, 1998.

[H] Herrlich, F.: Characteristic origamis. Preprint, Karlsruhe 2005.

[HL] Hubert, P., Lelièvre, S.: Prime arithmetic Teichmüller discs in H(2). To appear in Israel Journal of Mathematics. 
[HuSc] Hubert, P., Schmidt, T.: Invariants of translation surfaces. Annales de l'Institut Fourier 51 No. 2, 461-495 (2001).

[KK] Kuribayashi, A., Komiya,K.: On Weierstrass points and automorphisms of curves of genus three. Algebraic geometry, Proc. Summer Meet., Copenh. 1978, Lect. Notes Math. 732, 253-299 (1979).

[Lo] Lochak, P.: On arithmetic curves in the moduli space of curves. To appear in Journal of the Institute of Mathematics of Jussieu.

[McM] McMullen, C.: Billiards and Teichmüller curves on Hilbert modular surfaces. Journal of the American Mathematical Society 16 No. 4, 857-885 (2003).

[M1] Möller, M.: Teichmüller curves, Galois action and $\widehat{G T}$-relations. To appear in Math. Nachr.

[M2] Möller, M.: Shimura and Teichmüller curves. Preprint 2005, arXiv:math.AG/0501333

[S] Schmithüsen, G.: An algorithm for finding the Veech group of an origami. Experimental Mathematics 13, 459 - 472 (2004).

[W] Wolfart, J.: Werte hypergeometrischer Funktionen. Invent. math. 92, 187-216 (1988).

[V] Veech, W.A.: Teichmüller curves in moduli space, Eisenstein series and an application to triangular billiards. Invent. math. 97 No.3, 553-583 (1989). 